\title{
Angular dependence of the radiation power of a Josephson STAR-emitter
}

\author{
Richard A. Klemm ${ }^{1, *}$ and Kazuo Kadowaki ${ }^{2,0}$ \\ ${ }^{1}$ Department of Physics, University of Central Florida, Orlando, FL 32816, USA \\ ${ }^{2}$ Graduate School of Pure $\&$ Applied Sciences, University of Tsukuba, \\ 1-1-1, Tennodai, Tsukuba, Ibaraki 305-8573, Japan
}

(Dated: June 28, 2021)

\begin{abstract}
We calculate the angular dependence of the power of stimulated terahertz amplified radiation (STAR) emitted from a $d c$ voltage applied across a stack of intrinsic Josephson junctions. During coherent emission, we assume a spatially uniform $a c$ Josephson current density in the stack acts as a surface electric current density antenna source, and the cavity features of the stack are contained in a magnetic surface current density source. A superconducting substrate acts as a perfect magnetic conductor with $H_{\|, a c}=0$ on its surface. The combined results agree very well with recent experimental observations. Existing $\mathrm{Bi}_{2} \mathrm{Sr}_{2} \mathrm{CaCu}_{2} \mathrm{O}_{8+\delta}$ crystals atop perfect electric conductors could have Josephson STAR-emitter power in excess of $5 \mathrm{~mW}$, acceptable for many device applications.
\end{abstract}

PACS numbers: 07.57.Hm, 74.50.+r, 85.25.Cp

At present, broad-band terahertz $(\mathrm{THz})$ electromagnetic (EM) waves generated from femtosecond laser pulses and monochromatic $\mathrm{THz}$ waves generated by laser mixing, parametric resonance techniques, and quantum cascade lasers, etc., are the most common THz sources [1]. But these techniques are not cost effective in the "THz gap" region 0.1-10 THz required for many important applications. After many years of effort, by application of a static voltage across the intrinsic Josephson junctions in $\mathrm{Bi}_{2} \mathrm{Sr}_{2} \mathrm{CaCu}_{2} \mathrm{O}_{8+\delta}$ (BSCCO) mesas, coherent radiation power of $0.5 \mu \mathrm{W}$ was achieved 22]. The same technique on different samples since led to radiation power of $5 \mu \mathrm{W}$ and an output efficiency of $3 \times 10^{-4}[3]$. This is an exciting development, as the "THz gap" may soon be filled.

In both experiments, the onset of the intense, coherent $\mathrm{THz}$ radiation occurs in or near to the region in the current-voltage $(I-V)$ characteristic of negative differential resistance (NDR), as for the Gunn effect in Ge[4]. In the absence of more precise information, we assume that the stack of Josephson junctions acts partly as a cavity, in order to amplify the coherent radiation at the fundamental Josephson angular frequency $\omega_{J}$ and possibly its harmonics, and partly as a conductor with an $a c$ Josephson current, $J_{a c}\left(\boldsymbol{x}^{\prime}, t\right)=J\left(\boldsymbol{x}^{\prime}\right) \sum_{n=1}^{\infty} a_{n} \sin \left(n \omega_{J} t\right)$, where $\omega_{J}=2 e V_{0} /(N \hbar)$ is the Josephson angular frequency, $V_{0}$ is the $d c$ voltage applied across the coherent stack of $N \approx 10^{3}$ junctions, $e$ is the electric charge, $\hbar$ is the Planck constant divided by $2 \pi$, and $a_{n}$ is the relative amplitude of the $n$th harmonic of the intrinsic $a c$ Josephson current. The effective radiation sources at the mesa edges are respectively the surface magnetic current $\boldsymbol{M}_{S}$ arising from the electric field in the cavity generated by the non-uniform part of $J_{a c}\left(\boldsymbol{x}^{\prime}, t\right)[\underline{6}]$, and the surface electric current $\boldsymbol{J}_{S}$ arising from the uniform part of $J_{a c}\left(\boldsymbol{x}^{\prime}, t\right)$, as sketched in Fig. 1 (a). These sources are obtained from the Faraday and Ampère boundary conditions, respectively [5, [6], as used previously [7, 8, 9, 10, 11].

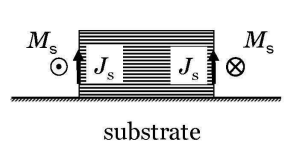

(a)

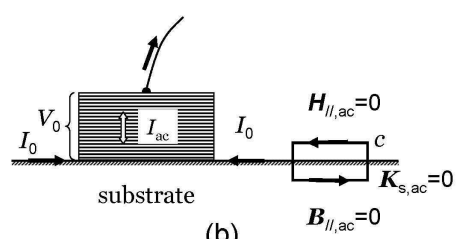

(b)
FIG. 1: (a) Sketch of a mesa with $\boldsymbol{J}_{S}$ and $\boldsymbol{M}_{S}$ surface current sources. (b) Mesa during coherent emission with applied $d c$ $I_{0}, V_{0}$, and $I_{a c}$ confined to it. Curve $c$ is the integration path for the Ampère law boundary condition. See text.

We first assume the mesa is suspended in vacuum. In Lorentz gauge, the vector potentials from the respective radiation sources are $[\underline{5},[\underline{6}$,

$$
\boldsymbol{A}(\boldsymbol{x}, t)=\sum_{n=1}^{\infty} e^{-i n \omega_{J} t} \frac{\mu_{0}}{4 \pi} \int \frac{d^{3} \boldsymbol{x}^{\prime} \boldsymbol{J}_{S n}\left(\boldsymbol{x}^{\prime}\right)}{R} e^{i k_{n} R},
$$

$R=\left|\boldsymbol{x}-\boldsymbol{x}^{\prime}\right|, k_{n}^{\prime}=n \omega_{J} \sqrt{\epsilon} / c=k_{n} \sqrt{\epsilon}$, inside and outside the mesa, $\epsilon$ is the dielectric constant of the mesa, $\boldsymbol{F}(\boldsymbol{x}, t)$ is given by $\boldsymbol{A}(\boldsymbol{x}, t)$ with $\epsilon_{0}$ and $\boldsymbol{J}_{S n}\left(\boldsymbol{x}^{\prime}\right)$ replaced with $\mu_{0}$ and $\boldsymbol{M}_{S n}\left(\boldsymbol{x}^{\prime}\right)$, respectively, and $\epsilon_{0}, \mu_{0}$, and $c$ are the vacuum dielectric constant, magnetic permeability, and speed of light, respectively; $\boldsymbol{J}_{S n}\left(\boldsymbol{x}^{\prime}\right)$ and $\boldsymbol{M}_{S n}\left(\boldsymbol{x}^{\prime}\right)$ are the electric and magnetic surface current densities corresponding to the $n$th harmonic of $J_{a c}\left(\boldsymbol{x}^{\prime}, t\right)$.

We first consider cylindrical mesas of radius $a \approx 50 \mu \mathrm{m}$ and height $h \approx 1 \mu \mathrm{m}$. In cylindrical $\left(\rho^{\prime}, \phi^{\prime}, z^{\prime}\right)$ coordinates, the sources $\boldsymbol{J}_{S n}\left(\boldsymbol{x}^{\prime}\right)$ and $\boldsymbol{M}_{S n}\left(\boldsymbol{x}^{\prime}\right)$ are

$$
\begin{aligned}
\boldsymbol{J}_{S n}\left(\boldsymbol{x}^{\prime}\right) & =\hat{\boldsymbol{z}}^{\prime} \frac{J_{J} a_{n}}{2} a \eta_{J}\left(z^{\prime}\right) \delta\left(\rho^{\prime}-a\right), \\
\boldsymbol{M}_{S n}\left(\boldsymbol{x}^{\prime}\right) & =\hat{\boldsymbol{\phi}}^{\prime} \delta_{n, 1} \frac{\tilde{E}_{0}}{2} a \cos \left(\phi^{\prime}-\phi_{0}\right) \eta_{M}\left(z^{\prime}\right) \delta\left(\rho^{\prime}-a\right),(3)
\end{aligned}
$$

where $\tilde{E}_{0}=E_{0} J_{1}\left(k_{1}^{\prime} a\right)$ is the effective electric field ampli- 
tude at the mesa edge, $k_{1}^{\prime} a=1.8412$ satisfies $J_{1}^{\prime}\left(k_{1}^{\prime} a\right)=0$ in order that $H_{\phi}\left(\rho^{\prime}=a\right)=0$ for the lowest transverse magnetic (TM) cylindrical cavity mode, $\mathrm{TM}_{110}^{z}[\underline{6}$, $J_{m}(x)$ is a standard Bessel function, and for no substrate, $\eta_{J}\left(z^{\prime}\right)=\eta_{M}\left(z^{\prime}\right)=\eta\left(z^{\prime}\right)=\Theta\left(z^{\prime}\right) \Theta\left(h-z^{\prime}\right)$, where $\Theta(x)$ is the Heaviside step function. The higher energy cavity mode energies are not integral multiples of one another [6]. Assuming the fundamental frequency of a non-uniform $J_{a c}\left(\boldsymbol{x}^{\prime}, t\right)$ excites a cavity mode, higher harmonics of it will not, so we take $\boldsymbol{M}_{S n} \propto \delta_{n, 1}$. Since $h<<a, r$ is the smallest length in the problem, $\eta\left(z^{\prime}\right) \rightarrow h \delta\left(z^{\prime}\right)$ for no substrate. Outside the mesa, we write $\boldsymbol{A}(\boldsymbol{x}, t)$ and $\boldsymbol{F}(\boldsymbol{x}, t)$ in spherical coordinates $(x, y, z)=r(\sin \theta \cos \phi, \sin \theta \sin \phi, \cos \theta)$. After averaging over $\phi_{0}$ and $t$, in the radiation zone $r>>a$ far from the mesa, the emitted power per unit solid angle is

$$
\begin{array}{r}
\frac{d P}{d \Omega} \underset{r / a \rightarrow \infty}{\rightarrow} \frac{Z_{0}\left(J_{J} v k_{1}\right)^{2}}{32 \pi^{2}}\left[\sin ^{2} \theta \sum_{n=1}^{\infty}\left|n a_{n} S_{n}^{J}(\theta) J_{0}\left(n k_{\theta}\right)\right|^{2}\right. \\
\left.+\alpha(\theta)\left(\cos ^{2} \theta J_{+}^{2}\left(k_{\theta}\right)+J_{-}^{2}\left(k_{\theta}\right)\right)\right], \quad \text { (4) }
\end{array}
$$

where $\alpha(\theta)=\frac{1}{2}\left|\tilde{E}_{0} S_{1}^{M}(\theta)\right|^{2} /\left(Z_{0} J_{J}\right)^{2}, J_{ \pm}(z)=\left[J_{2}(z) \pm\right.$ $\left.J_{0}(z)\right] / 2, k_{\theta}=k_{1} a \sin \theta, Z_{0}=\sqrt{\epsilon_{0} / \mu_{0}}$ is the vacuum impedance, $S_{n}^{M}(\theta)=S_{n}^{J}(\theta)=1$ for no substrate, and the details are presented elsewhere[12]. In Fig. 2, plots for no substrate of the intensity $I(\theta) \propto d P(\theta) / d \Omega$ versus $\theta$ at the fundamental with $k_{1} a=1.8412 / \sqrt{\epsilon}$ from separate $\boldsymbol{M}_{S 1}$ and $\boldsymbol{J}_{S}$ sources are shown by curves (A) and (B), respectively, and the combined fundamental output with $\alpha(0)=0.6$ is shown by curve (C). $I(\theta)$ at the second $a c$ Josephson current harmonic with $k_{2}=2 k_{1}$ is shown by curve (E).

We now consider a rectangular mesa of width $w$, length $\ell$, and height $h$ with no substrate. To fit experiment, we assume $\mathrm{TM}_{n 00}^{z}$ modes, which oscillate in position with integral multiples of half-wavelengths along the mesa widths [2, 8, 9, 10]. In rectangular source coordinates $\left(x^{\prime}, y^{\prime}, z^{\prime}\right)$,

$$
\begin{aligned}
\boldsymbol{J}_{S n}\left(\boldsymbol{x}^{\prime}\right)= & \hat{\boldsymbol{z}}^{\prime} \frac{J_{J} a_{n}}{4} \eta_{J}\left(z^{\prime}\right) \sum_{\sigma= \pm}\left[f_{\sigma}\left(x^{\prime}, y^{\prime}\right)+g_{\sigma}\left(x^{\prime}, y^{\prime}\right)\right] \\
\boldsymbol{M}_{S n}\left(\boldsymbol{x}^{\prime}\right)= & \frac{\tilde{E}_{0 n}}{4} \eta_{M}\left(z^{\prime}\right) \sin \left[n\left(x^{\prime}-x_{n}\right) \pi / w\right] \\
& \times \sum_{\sigma= \pm} \sigma\left[\hat{\boldsymbol{y}}^{\prime} f_{\sigma}\left(x^{\prime}, y^{\prime}\right)-\hat{\boldsymbol{x}}^{\prime} g_{\sigma}\left(x^{\prime}, y^{\prime}\right)\right] \\
f_{\sigma}\left(x^{\prime}, y^{\prime}\right)= & w \delta\left(x^{\prime}+\sigma w / 2\right) \Theta\left[(\ell / 2)^{2}-\left(y^{\prime}\right)^{2}\right] \\
g_{\sigma}\left(x^{\prime}, y^{\prime}\right)= & \ell \delta\left(y^{\prime}+\sigma \ell / 2\right) \Theta\left[(w / 2)^{2}-\left(x^{\prime}\right)^{2}\right]
\end{aligned}
$$

where the $\mathrm{TM}_{n 00}^{z}$ cavity mode energy is degenerate for $-w / n \leq x_{n} \leq w / n$.

We treat $\boldsymbol{M}_{S n}$ in two models. In Model I, the average output power is taken to be $\left\langle P\left(x_{n}\right)\right\rangle_{I}=$ $\frac{1}{2}[P(0)+P(w / n)][\underline{6}]$. In Model II, we let $\left\langle P\left(x_{n}\right)\right\rangle_{I I}=$

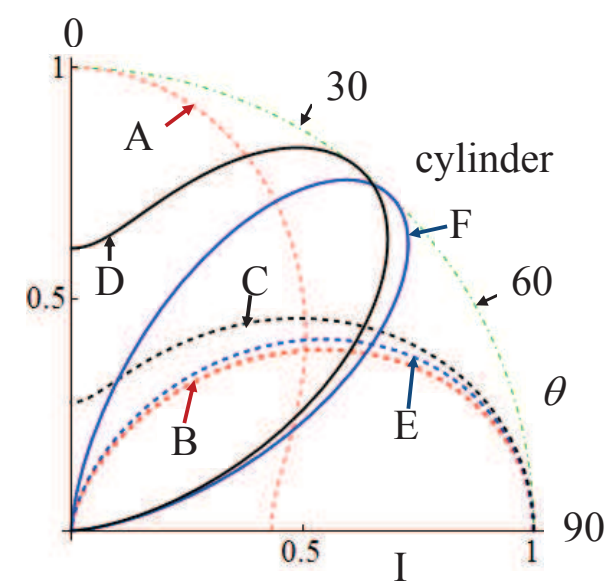

FIG. 2: (color online) Polar plots of the radiation zone intensities $I(\theta)$ from Eq. (4) in arbitrary units versus $\theta$ (degrees) of magnetic and electric source fundamental outputs $[(\mathrm{A}),(\mathrm{B})]$ and the combined output with $\alpha(0)=0.6$ with no $[(\mathrm{C}),(\mathrm{E})]$ and superconducting $[(\mathrm{D}),(\mathrm{F})]$ substrates, of the fundamental with $k_{1}^{\prime} a=1.8412$ and the second $a c$ Josephson current harmonic, respectively, with $k_{2}=2 k_{1}$ for a cylindrical mesa.

$\int_{-w / n}^{w / n} d x_{n} P\left(x_{n}\right) n / 2 w$. Then, in spherical coordinates, the time-averaged power per unit solid angle in the radiation zone is

$$
\begin{gathered}
\frac{d P}{d \Omega} \underset{r / a \rightarrow \infty}{\rightarrow} \frac{Z_{0}\left(J_{J} \tilde{v} k_{1}\right)^{2}}{128 \pi^{2}} \sum_{n=1}^{\infty} n^{2}\left[\left|\sin \theta a_{n} \chi_{n} S_{n}^{J}(\theta)\right|^{2}\right. \\
+\alpha_{n}(\theta)\left(C_{n}^{i}+D_{n}^{i}-\sin ^{2} \theta\left[C_{n}^{i} \cos ^{2} \phi\right.\right. \\
\left.\left.\left.+D_{n}^{i} \sin ^{2} \phi-E_{n}^{i} \sin \phi \cos \phi\right]\right)\right] \\
\chi_{n}=\quad \cos X_{n} \frac{\sin Y_{n}}{Y_{n}}+\cos Y_{n} \frac{\sin X_{n}}{X_{n}}
\end{gathered}
$$

$X_{n}=\left(k_{n} w / 2\right) \sin \theta \cos \phi, Y_{n}=\left(k_{n} \ell / 2\right) \sin \theta \sin \phi$, where $i=\mathrm{I}, \mathrm{II}, \alpha_{n}(\theta)=\left|\tilde{E}_{0 n} S_{n}^{M}(\theta)\right|^{2} /\left(2 Z_{0} J_{J}\right)^{2}, \tilde{v}=w \ell h$, and the $C_{n}^{i}(\theta, \phi), D_{n}^{i}(\theta, \phi)$, and $E_{n}^{i}(\theta, \phi)$ are given elsewhere 12.

Plots of $I(\theta, 0) \propto d P(\theta, 0) / d \Omega$ in arbitrary units versus $\theta$ in degrees at $\phi=0$ from Eq. (9) with $S_{n}^{J}(\theta)=$ $S_{n}^{M}(\theta)=1, \ell=20 w / 3, k_{1}^{\prime}=\pi / w=k_{1} \sqrt{\epsilon}, k_{2}=2 k_{1}$ are given by curves (A) and (B) in Fig. 3[12]. In experiment [3], the maximum fundamental $I(\theta, 0)$ is generally at $\theta_{\max } \approx 30-40^{\circ}$, and $I\left(90^{\circ}, 0\right)=0$. Although curve (A), obtained from $\boldsymbol{M}_{S 1}$ alone, yields $I\left(90^{\circ}, 0\right)=0$, it has $\theta_{\max }=0$. Hence, it is necessary to add the $\boldsymbol{J}_{S 1}$ source. Curve (B) is obtained for $\alpha(0)=0.20,0.40$ for $i=\mathrm{I}$ and II, respectively. It has $\theta_{\max } \approx 40^{\circ}$, nearly as observed, but also yields a large $I\left(90^{\circ}, 0\right)$ value, unlike the observations. The corresponding second harmonic intensity is shown in curve $(\mathrm{C})$, which also shows $I\left(90^{\circ}, 0\right) \neq 0$, unlike present experiments 3 . This unexpected combination of $I\left(90^{\circ}, 0\right)=0$ and $\theta_{\max } \approx 30^{\circ}$ led us to consider the effects of existing BSCCO substrates [2, 3]. As 


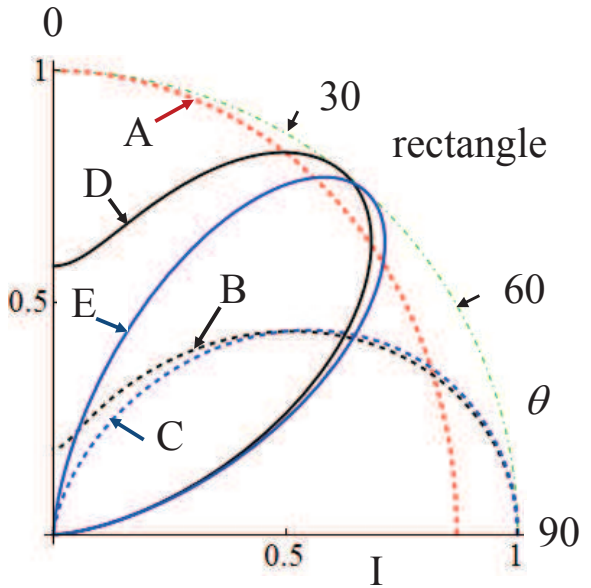

FIG. 3: (color online) Polar plots of the radiation zone intensities $I(\theta, 0)$ from Eq. (9) in arbitrary units versus $\theta$ (degrees) with no [(A) $M_{S}$ source only, (B) combined, (C) $n=2$, Model II] and superconducting [(D),(E), $n=2$, Model I] substrates, of the fundamental with $k_{1}^{\prime} w=\pi$ and the second harmonic, respectively, with $k_{2}^{\prime} w=2 \pi$ normal to the length $(\phi=0)$ of a rectangular mesa with $\ell=20 w / 3$.

sketched in Fig. 1 (b), during coherent Josephson radiation, the $a c$ Josephson current is essentially confined to the mesa. With only a $d c$ surface current density $\propto I_{0}$ and $B_{\|, a c}(t)=0$ beyond the skin depth $(\approx 0.15 \mu \mathrm{m}$ ) inside the BSCCO substrate due to the $a c$ Meissner effect, the Ampére-Maxwell boundary condition forces $H_{\|, a c}(t)=0$ just above the BSCCO substrate[5]. This corresponds to a perfect magnetic conductor (PMC) substrate, with the effective image sources opposite to those of a PEC substrate [6, 12]. Thus, for a BSCCO substrate, we replace $\eta_{J}\left(z^{\prime}\right)$ and $\eta_{M}\left(z^{\prime}\right)$ in Eqs. (2) and (5) by

$$
\eta_{-}\left(z^{\prime}\right)=\eta\left(z^{\prime}\right)-\eta\left(-z^{\prime}\right)=\operatorname{sgn}\left(z^{\prime}\right) \Theta\left[h^{2}-\left(z^{\prime}\right)^{2}\right] .
$$

In the radiation zone, $h<<a, r$ and $h<<w, \ell, r$ for both mesa types, so we assume $h \ll 1 / k_{n}$ for the relevant $n$. Expanding $e^{i k_{n} R} / R$ in Eq. (1) for small $z^{\prime}$,

$$
S_{n}^{J}(\theta)=S_{n}^{M}(\theta) \underset{r \rightarrow \infty}{\rightarrow}-i k_{n} h \cos \theta \Theta\left(90^{\circ}-\theta\right) .
$$

Plots of $I(\theta)$ in the radiation zone for a cylindrical mesa with $k_{1} a=1.8412 / \sqrt{\epsilon}$ and $k_{2}=2 k_{1}$ atop a superconducting substrate are shown by curves (D) and (F) in Fig. 2. Near $90^{\circ}$, a superconducting substrate has a drastic effect on the power emitted from the $J_{S}$ source. Plots of $I(\theta, 0)$ for a rectangular mesa on a superconducting substrate in the radiation zone of the fundamental in both models and of the second harmonic in Model II, are shown respectively by curves (D) and (E) in Fig. 3. Since intense higher harmonics can arise only from the $\boldsymbol{J}_{S n}$ in cylindrical mesas, the study of cylindrical mesas could provide valuable information regarding the primary radiation source.
Preliminary experimental fundamental $I(\theta, 0)$ results for mesas with $w=60 \mu \mathrm{m}, \ell=400 \mu \mathrm{m}$, and $h=1 \mu \mathrm{m}$ are in good agreement with curve (D) shown in Fig. $3[3$. The effect of a superconducting substrate is crucial for $\theta \approx 90^{\circ}$ (nearly parallel to the substrate), as shown in Figs. 2 and 3. More importantly, a superconducting substrate has a drastic effect upon the radiation power. Since $k_{1}=\pi / w$ for rectangular mesas [3] at the fundamental $\theta_{\max }, 40^{\circ}$ from curve (B) in Fig. 3, $\left|S_{1}^{J, M}\left(\theta_{\max }\right)\right|^{2}=\left[h k_{1} \cos \left(40^{\circ}\right)\right]^{2} \approx 1.6 \times 10^{-3}$. Hence, replacing the superconducting substrate by an insulating one could enhance the power output by 600 . Replacing it by a standard PEC could further quadruple it [6, 12]. Since output power of $5 \mu \mathrm{W}$ was achieved for rectangular mesas on superconducting substrates [3] , coherent $\mathrm{THz}$ radiation power in excess of $5 \mathrm{~mW}$, acceptable for many applications, might be attainable from existing BSCCO samples. Since the frequency range of the coherent radiation lies between those of a maser and a laser, we hereby declare the device to be a Josephson STAR-emitter, for stimulated terahertz amplified radiation emitter.

We thank X. Hu, S. Lin, B. Markovic, N. F. Pedersen, and M. Tachiki for stimulating discussions. This work was supported in part both by the JST (Japan Science and Technology Agency) CREST project, by the WPI Center for Materials Nanoarchitechtonics (MANA), by the JSPS (Japan Society for the Promotion of Science) CTC program and by the Grant-in Aid for Scientific Research (A) under the Ministry of Education, Culture, Sports, Science and Technology (MEXT) of Japan. R.A.K. would also like to thank the University of Tsukuba for its kind hospitality.

* Electronic address: klemm@physics.ucf.edu

$\dagger$ Electronic address: kadowaki@ims.tsukuba.ac.jp

[1] For a review, M. Tonouchi, Nature Photon. 1, 97 (2007).

[2] L. Ozyuzer et al., Science 318, 1291 (2007).

[3] K. Kadowaki et al., unpublished.

[4] J. Millman and C. C. Halkias, Electronic Devices and Circuits, (McGraw-Hill, Tokyo, 1967).

[5] J. D. Jackson, Classical Electrodynamics (Wiley, NY, third edition, 1999).

[6] C. A. Balanis, Antenna Theory, Analysis and Design (Wiley, Hoboken, NJ, third edition, 2005). See Fig. 4.13.

[7] H. Matsumoto, T. Koyama, and M. Machida, Physica C (2008), doi:10.1016/j.physc.2007.11.030

[8] L. N. Bulaevskii and A. E. Koshelev, J. Supercond. and Novel Magn. 19, 349 (2006).

[9] L. N. Bulaevskii and A. E. Koshelev, Phys. Rev. Lett. 99, 057002 (2007).

[10] A. E. Koshelev and L. N. Bulaevskii, arXiv:0708.3269v2.

[11] S. Lin and X. Hu, Phys. Rev. Lett. 100, 247006 (2008).

[12] R. A. Klemm and K. Kadowaki, arXiv:0807.3082. 